\title{
La gestion de la pandémie dans le système scolaire allemand
}

\section{Werner Zettelmeier}

\section{OpenEdition}

1 Journals

Édition électronique

URL : https://journals.openedition.org/ries/10303

DOI : 10.4000/ries. 10303

ISSN : 2261-4265

Éditeur

France Education international

Édition imprimée

Date de publication : 1 avril 2021

Pagination : 23-26

ISBN : 978-2-85420-629-6

ISSN : 1254-4590

Référence électronique

Werner Zettelmeier, " La gestion de la pandémie dans le système scolaire allemand 》, Revue internationale d'éducation de Sèvres [En ligne], 86 | avril 2021, mis en ligne le 01 avril 2022, consulté le 25 mars 2023. URL : http://journals.openedition.org/ries/10303 ; DOl : https://doi.org/10.4000/ries. 10303

Ce document a été généré automatiquement le 25 mars 2023.

Tous droits réservés 


\title{
La gestion de la pandémie dans le système scolaire allemand
}

\author{
Werner Zettelmeier
}

1 Depuis le début de la pandémie mondiale liée à la Covid-19, la gestion de cette crise inédite par les pouvoirs publics fait l'objet de débats parfois vifs en Allemagne. En effet, les responsables politiques se voient contraints de trouver un équilibre difficile à établir entre, d'une part, la préservation des libertés individuelles à laquelle ils sont tenus par un État démocratique fondé sur le respect des droits civiques et, d'autre part, la nécessité de restreindre certains de ces mêmes droits pour lutter contre la progression de la pandémie et ses conséquences en termes de santé publique. La proportionnalité et la légitimation démocratique de ces restrictions relèvent d'une importance fondamentale pour le fonctionnement d'un État de droit, de même que pour leur acceptation par les citoyens et, par conséquent, pour leur efficacité.

2 Le système éducatif et sa résilience représentent un domaine particulièrement sensible dans la gestion de la crise sanitaire. Dans les pays de l'Union européenne, on constate en effet que les mesures prises dès mars 2020, et notamment la fermeture des établissements scolaires et universitaires, ont suscité un débat sur les conséquences possibles du basculement abrupt vers un enseignement basé sur les technologies modernes de communication. La recherche empirique en éducation a démontré pour nombre de pays européens, dont l'Allemagne et la France, un lien très fort entre la réussite scolaire et l'origine sociale des élèves. Des voix se sont élevées dans les deux pays pour dire que l'enseignement en ligne risquait de renforcer des inégalités sociales déjà existantes avant la crise et de provoquer la démotivation et le décrochage des élèves issus des milieux socialement moins favorisés.

3 De plus, en Allemagne, le débat sur les mesures prises par les pouvoirs publics pour gérer la crise sanitaire dans le système éducatif a non seulement porté sur les problèmes techniques, organisationnels et pédagogiques soulevés par le passage à un enseignement en ligne, mais il a très vite relancé des polémiques en quelque sorte indissociables du système éducatif, à savoir son organisation fédérale. 


\section{Le fédéralisme : un élément constitutif de l'ordre constitutionnel allemand}

4 Il convient de rappeler quelques aspects institutionnels de cet élément constitutif de l'ordre constitutionnel allemand. Ils permettent de mieux comprendre les problèmes rencontrés par le système éducatif dans la gestion de la crise sanitaire.

5 L'enseignement scolaire est probablement la politique publique la plus connue des Allemands, car vécue par eux dès leur plus jeune âge, et pour laquelle les Länder disposent d'une compétence législative et réglementaire quasi exclusive. Le gouvernement fédéral n'a donc que des compétences d'appui très limitées, du moins pour ce qui est des affaires scolaires. Les Länder créent eux-mêmes la loi et les normes juridiques dans ce domaine. Ils disposent d'une " qualité étatique » et contribuent ainsi à une diversité certaine dans les politiques scolaires menées. Cela va de l'organisation de l'année scolaire jusqu'à la définition des missions de l'école, en passant par la gestion des enseignants (y compris universitaires), qui n'ont pas de statut national mais dépendent $\mathrm{du}$ Land, et la définition des programmes d'enseignement, des modalités d'évaluation, des diplômes et des types d'établissements. Cette situation rend nécessaire une concertation permanente et systématique entre les seize Länder - égaux en droits - sur les décisions en matière de politique éducative, afin que l'autonomie dont ils disposent dans ce domaine reste compatible avec une autre obligation, également inscrite dans la Loi fondamentale, qui est celle de préserver l'équité des conditions de vie sur le territoire allemand. Dans la recherche de l'équilibre entre les deux impératifs constitutionnels, il s'est créé au cours de l'histoire depuis 1949 - entre les seuls Länder ou entre les deux niveaux de l'organisation de l'État - un système de plus en plus complexe de concertation et de régulation, à commencer par la Conférence permanente des ministres en charge des affaires d'éducation (Kultusministerkonferenz: KMK) créée dès 1946, soit avant même la naissance de la République fédérale. Afin d'éviter des situations de blocage, ce système, appelé « fédéralisme coopératif » par les spécialistes, oblige les acteurs à rechercher en permanence un compromis entre des avis parfois très divergents selon les majorités politiques au pouvoir dans les différents Länder ou entre Länder et gouvernement fédéral. Même si la KMK s'abstient de formuler des recommandations détaillées afin de laisser une marge de manœuvre dans leur mise en œuvre, ces dernières, à défaut d'avoir une valeur législative en droit - les parlements régionaux doivent transposer ces recommandations dans leur législation respective - ont des effets normatifs de fait très importants car le compromis trouvé est souvent le plus petit dénominateur commun. Ce mode de fonctionnement de la KMK oblige les Länder à respecter une approche collective dans la résolution des problèmes d'ordre national et à ne pas trop s'écarter d'un compromis possible par des démarches isolées. Ainsi la décision de la KMK, prise le 25 mars 2020, de maintenir - en décalant de quelques semaines après la fin du premier confinement - les épreuves écrites du baccalauréat sur tout le territoire allemand, fait suite à une proposition, non concertée, du ministère en charge des affaires scolaires du Land de Schleswig-Holstein de renoncer à ces épreuves face à la progression du virus et de délivrer le diplôme sur la base des résultats du contrôle continu obtenus par les candidats durant les deux dernières années de scolarité. Les épreuves écrites ont eu lieu finalement dans toute l'Allemagne et la KMK a motivé sa décision par la nécessité d'assurer l'équité des 
conditions d'obtention d'un diplôme sur le territoire et de préserver la reconnaissance de sa valeur ${ }^{1}$.

\section{La gestion de la pandémie dans les écoles : entre approbation et critique}

6 La lenteur du processus de prise de décision, ainsi que l'absence d'un contrôle parlementaire direct de ces instances «intergouvernementales» de concertation sont cependant régulièrement critiquées par les partisans d'une certaine recentralisation de la politique éducative. La médiatisation des démarches, parfois très différentes, adoptées par les Länder dans la crise sanitaire et leur instrumentalisation dans le débat politique pour dénoncer les blocages institutionnels par rapport à d'autres pays ont alimenté ces critiques de longue date. Ainsi, le président du Deutscher Lehrerverband ${ }^{2}$ a reproché au fédéralisme allemand d'avoir fait " piètre figure ${ }^{3}$ » dans sa gestion de la crise du coronavirus, tout en se disant attaché aux prérogatives des Länder en matière d'éducation. Sa critique porte essentiellement sur la lenteur et la complexité des décisions de la KMK - il se prononce en faveur de l'abandon des votes à l'unanimité - et sur l'équipement informatique, jugé encore insuffisant, des établissements, des enseignants et des élèves.

7 Dans la gestion de la crise sanitaire par le système éducatif, le gouvernement fédéral ne dispose donc pratiquement d'aucune compétence; toute annonce du gouvernement fédéral dans ce domaine - telle la fermeture des établissements scolaires et universitaires le 16 mars 2020 - suppose une concertation préalable entre les Länder au sein de la KMK sous forme de recommandation ${ }^{4}$, ainsi qu'entre le chef du gouvernement fédéral et les ministres-présidents des seize Länder pour les mesures de protection à adopter. La concertation entre les acteurs aux deux niveaux de l'État est également nécessaire dans le domaine de la politique de santé. En effet, la quasi-totalité des politiques publiques pour lesquelles le niveau fédéral dispose d'une compétence constitutionnelle sont mises en œuvre par les administrations des Länder, le gouvernement fédéral ne disposant d'une administration propre déconcentrée que dans des domaines - énumérés de façon limitative par la Loi fondamentale - pour lesquels il a une compétence exclusive (l'armée et le service diplomatique notamment). Cette approche consensuelle de la crise sanitaire, s'appuyant sur une large concertation avec les Länder, mais aussi entre les deux grands partis historiques ${ }^{5}$ de la scène politique allemande qui forment le gouvernement fédéral actuel, ainsi que sur les avis d'instituts de recherche tels que l'Institut Robert-Koch de Berlin (RKI), qui est l'agence fédérale de santé, largement plébiscités par les personnels de santé, ont permis aux pouvoirs publics allemands de bénéficier d'une acceptation assez large - du moins dans un premier temps et jusqu'au reconfinement de l'automne 2020 - des restrictions des libertés individuelles. À ce titre, une étude d'un groupe de chercheurs de l'Université de Mannheim livre des résultats intéressants sur l'évolution de l'approbation par la population des différentes mesures prises et/ou discutées par les pouvoirs publics entre mars et juillet 2020. Un échantillon représentatif de 3600 personnes a été interrogé systématiquement toutes les semaines pendant cette période. Si la fermeture des établissements scolaires et universitaires a recueilli un taux d'approbation très élevé au début (plus de $90 \%$ ), c'est cette mesure qui a perdu le plus rapidement et massivement 
en approbation, car le taux s'est stabilisé à partir de fin mai 2020 à un peu moins de $25 \%$, l'un des taux les plus bas recueillis parmi toutes les mesures questionnées ${ }^{6}$.

Dans l'analyse des faiblesses de l'organisation fédérale par rapport à une organisation étatique centralisée, plusieurs arguments sont avancés depuis le début de la crise sanitaire : la lenteur de la prise de décision, la division du territoire national en entités gérées différemment du point de vue du droit, ce qui augmenterait l'illisibilité de l'action politique, le risque étant que les décideurs politiques instrumentalisent cette diversité à des fins de communication stratégique au détriment d'approches plus collectives et concertées. Ces arguments ne sont pas nouveaux et accompagnent l'évolution du fédéralisme allemand depuis ses origines. On peut y opposer les arguments en faveur d'une prise de décision décentralisée qui, elle aussi, a trouvé dans la pandémie sa justification. Le fédéralisme allemand comporte, depuis sa refondation après 1945, des éléments de coopération et de concurrence, mais il n'a jamais été un fédéralisme à l'américaine visant à une séparation stricte des compétences entre le niveau fédéral et celui des Länder. L'obligation constitutionnelle d'assurer l'équité des conditions de vie a limité efficacement, somme toute, y compris dans les périodes de crise, le risque d'une atomisation du droit sur le territoire national et oblige tous les acteurs à trouver un équilibre entre des décisions centrales, mais négociées et concertées avec une multitude d'acteurs, et des décisions individuelles prises au niveau décentralisé. La gestion décentralisée a finalement su s'adapter aux situations spécifiques locales et répondre en cela à l'attente exprimée par la population, à savoir une production de biens publics efficace, en phase avec les besoins du terrain et dans des délais raisonnables. En même temps, il faut préserver au mieux les libertés individuelles par l'implication dans la prise de décision d'un maximum d'acteurs, afin d'empêcher une concentration de tous les pouvoirs en un seul lieu. Dans le cas concret de la pandémie et de la gestion de ses conséquences par le système scolaire allemand, les mécanismes de concertation, devenus peut-être trop routiniers avec le temps, ont été mis à l'épreuve et vont devoir évoluer. En outre, les Länder devront aller au-delà d'une concertation sur les dates de confinement ou de déconfinement, afin de permettre une réflexion plus pédagogique au niveau national sur les avantages et les limites de l'enseignement en ligne, de fixer les critères d'une offre cohérente de formation des enseignants en la matière, et de mettre à disposition des établissement les outils numériques requis.

\section{NOTES}

1. Il est à noter que les épreuves du baccalauréat en Allemagne, qui n'est pas un examen centralisé au niveau national, sont organisées selon un calendrier propre à chaque Land entre fin janvier et juin de chaque année. 
2. Le Deutscher Lehrerverband revendique quelque 165000 adhérents sur les 830000 enseignants que compte l'enseignement scolaire allemand. Il convient de préciser que les Kindergärten (écoles maternelles) relèvent de la compétence des ministères régionaux des affaires sociales. Les personnels qui y travaillent n'ont ni le statut, ni la formation d'enseignant, ils/elles sont éducateurs/éducatrices.

3. FAZ.net, 21 janvier 2021.

4. Dans une décision du 12 mars 2020, les ministres en charge des affaires scolaires des 16 Länder se sont accordés sur le principe d'une démarche concertée et conjointe dans les mesures à prendre face au virus.

5. Parmi les partis politiques représentés au Bundestag, ceux de l'opposition, les Verts, Die Linke (gauche radicale) et les Libéraux, ont approuvé globalement cette démarche; seul le parti populiste AfD (Alternative pour l'Allemagne) s'en est clairement écarté en apportant son soutien au mouvement "anti-masques » et aux manifestations spectaculaires organisées par ce dernier dans quelques grandes villes allemandes durant l'été 2020 .

6. Blom A. (2020). «Zum gesellschaftlichen Umgang mit der Corona-Pandemie. Ergebnisse der Mannheimer Corona-Studie ». Aus Politik und Zeitgeschichte, $n^{\circ}$ 35-37, p. 16-22.

INDEX

Index géographique : Allemagne

Palabras claves : crisis sanitaria, política educacional, política pública, éxito escolar

Keywords : health crisis, educational policy, public policy, academic success

Mots-clés : crise sanitaire, politique éducative, politique publique, réussite scolaire

\section{AUTEUR \\ WERNER ZETTELMEIER}

Université de Cergy-Pontoise 